\title{
Add-On Sitagliptin Therapy for Insulin-Treated Type 2 Diabetes: An Analysis of Hemoglobin A1c and Other Variables Using ASSIST-K Follow-Up Data
}

\author{
Masashi Ishikawa ${ }^{a}$, Masahiko Takai ${ }^{a}$, Hajime Maeda ${ }^{a}$, Akira Kanamori ${ }^{\text {a }}$, Akira Kubota ${ }^{\mathrm{a}}$, Hikaru Amemiya ${ }^{\mathrm{a}}$, \\ Takashi Iizuka ${ }^{a}$, Kotaro Iemitsu ${ }^{a}$, Tomoyuki Iwasaki ${ }^{a}$, Goro Uehara ${ }^{a}$, Shinichi Umezawa ${ }^{a}$, Mitsuo Obana ${ }^{a}$, \\ Hideaki Kaneshige ${ }^{a}$, Mizuki Kaneshiro ${ }^{a}$, Takehiro Kawata ${ }^{a}$, Nobuo Sasai ${ }^{a}$, Tatsuya Saito ${ }^{\text {a }}$ Tetsuo Takuma ${ }^{a}$, \\ Hiroshi Takeda ${ }^{a}$, Keiji Tanaka ${ }^{a}$, Shigeru Nakajima ${ }^{a}, K_{\text {Kazuhiko Hoshino }}{ }^{\text {a }}$, Shin Honda ${ }^{a}$, Hideo Machimura ${ }^{a}$, \\ Kiyokazu Matoba ${ }^{a}$, Fuyuki Minagawa ${ }^{a}$, Nobuaki Minamia, Yukiko Miyairia, Atsuko Mokubo ${ }^{a}$, \\ Tetsuya Motomiya ${ }^{\mathrm{a}}$, Manabu Waseda ${ }^{\mathrm{a}}$, Masaaki Miyakawa ${ }^{\mathrm{a}}$, Yasuo Terauchi ${ }^{\mathrm{b}}$, \\ Yasushi Tanaka ${ }^{c}$, Ikuro Matsuba ${ }^{a}$, , e
}

\begin{abstract}
Background: Sitagliptin was the first dipeptidyl peptidase-4 (DPP-4) inhibitor approved in Japan. Its efficacy and safety have been demonstrated, both as monotherapy and in combination with oral antidiabetic agents or insulin. However, reduction of hemoglobin A1c (HbA1c) by sitagliptin is insufficient in some patients. Therefore, data from an observational study of sitagliptin as add-on therapy to insulin in patients with type 2 diabetes (ASSIST-K) were used to conduct factor analysis of the 12-month changes in $\mathrm{HbAlc}$, body weight, estimated glomerular filtration rate (eGFR), and adverse events (AEs).

Methods: At member institutions of Kanagawa Physicians Association specializing in diabetes, outpatients with type 2 diabetes receiving insulin were followed for 12 months after addition of sitagliptin. The HbA1c (National Glycohemoglobin Standardization Program), blood glucose (fasting/postprandial), body weight, eGFR, and AEs were evaluated at each specified time. Multivariate analysis was performed by using sex and age as explanatory variables and the following response variables: the change in $\mathrm{HbAlc}$, body weight, or eGFR after 12 months of sitagliptin treatment, and occurrence of AEs.
\end{abstract}

Results: Of 1,168 patients registered in the ASSIST-K study, 412 patients were included in this analysis, excluding those not receiv-

Manuscript submitted November 27, 2018, accepted December 10, 2018

aStudy Group of the Diabetes Committee, Kanagawa Physicians Association, Kanagawa, Japan

${ }^{b}$ Department of Endocrinology and Metabolism, Yokohama City University, Kanagawa, Japan

'Division of Metabolism and Endocrinology, Department of Internal Medicine, St. Marianna University School of Medicine, Kanagawa, Japan

dMatsuba Medical Clinic, Kanagawa, Japan

${ }^{e}$ Corresponding Author: Ikuro Matsuba, Matsuba Medical Clinic, 2-159 Tsukagoshi, Saiwai-ku, Kawasaki-shi, Kanagawa 212-0024, Japan.

Email: ikuro@matsuba-web.com

doi: https://doi.org/10.14740/jem540 ing insulin before sitagliptin, those in whom the 12-month change in $\mathrm{HbAlc}$ could not be calculated, and those with missing data on explanatory variables. There was a significant decrease in HbA1c and eGFR, but no significant change in body weight. AEs observed in $>$ 10 patients were severe hypoglycemia (14 patients, 3.4\%) and constipation (13 patients, 3.2\%). Factor analysis revealed the following points: 1) Concurrent dyslipidemia and baseline HbA1c influenced the 12-month change in HbA1c; 2) Baseline body mass index and HbA1c influenced the 12-month change in body weight; and 3) Concurrent dyslipidemia, baseline sulfonylurea treatment, baseline body mass index, and baseline eGFR influenced the 12-month change in eGFR. In addition, the risk of severe hypoglycemia or constipation was significantly influenced by baseline HbAlc.

Conclusions: Patients with type 2 diabetes showing higher HbA1c levels after add-on sitagliptin therapy had concurrent dyslipidemia and a lower baseline HbAlc. Severe hypoglycemia or constipation was more likely to occur in patients with a low baseline HbAlc.

Keywords: Type 2 diabetes; Dipeptidyl peptidase-4 inhibitor; Sitagliptin; Hemoglobin A1c

\section{Introduction}

Dipeptidyl peptidase-4 (DPP-4) inhibitors are a class of oral hypoglycemic agents (OHAs) that selectively inhibit DPP-4, an enzyme involved in metabolism of incretins (glucagon-like peptide-1 and glucose-dependent insulinotropic polypeptide), thus promoting insulin secretion in a glucose-dependent manner by elevation of endogenous incretin levels [1, 2]. In 2017, a total of nine DPP-4 inhibitors were marketed in Japan, including two agents that only require once-weekly administration $[3,4]$. Two previous meta-analyses have not identified any significant differences among DPP-4 inhibitors with regard to improvement in glycemic control $[5,6]$ and these agents are characterized by a low risk of causing hypoglycemia or weight 
gain [7].

Sitagliptin was the first DPP-4 inhibitor released in Japan [8]. Although its efficacy has been confirmed as monotherapy and in combination with oral antidiabetic agents or insulin [9], significant reduction of hemoglobin Alc (HbAlc) is not achieved by sitagliptin treatment in some patients.

To confirm efficacy and safety in the routine clinical setting, we previously investigated outcomes at 1 year after initiation of sitagliptin therapy in patients with type 2 diabetes and poor glycemic control who were being managed by diabetologists. We have reported the results for patients not using insulin (ASSET-K study) [10-15], as well as the findings obtained in patients using insulin (ASSIST-K study) [16-18]. A similar investigation was also conducted in non-insulin-using patients with type 2 diabetes attending medical institutions not specializing in diabetes (ATTEST-K study), after which data from these three studies were combined for factor analysis of the changes in $\mathrm{HbA} 1 \mathrm{c}$, body weight, and estimated glomerular filtration rate (eGFR) up to 12 months of sitagliptin therapy [19].

In the present study, we conducted factor analysis of data from the ASSIST-K study to investigate the changes in $\mathrm{HbAlc}$, body weight, eGFR, and adverse events (AEs) over 12 months after initiation of sitagliptin in insulin-treated patients with type 2 diabetes and poor glycemic control who were attending medical institutions that specialized in managing diabetes.

\section{Methods}

\section{Study design}

The ASSIST-K study was a 12-month multicenter observational study performed at medical institutions specializing in the management of diabetes that were affiliated with Kanagawa Physicians Association.

\section{Patients}

The subjects were patients over 20 years old with type 2 diabetes attending the outpatient clinics of institutions affiliated with Kanagawa Physicians Association who started sitagliptin treatment because of poor glycemic control after receiving insulin for at least 1 month. The following exclusion criteria were employed: 1) Patients with a history of hypersensitivity to any component of sitagliptin; 2) Patients with a history of severe ketoacidosis, diabetic coma, or precoma within 6 months before initiation of sitagliptin; 3) Patients with severe infection or severe trauma; 4) Patients in the perioperative period before and after surgery; 5) Patients on treatment with glinides; and 6) Other patients judged to be inappropriate by the attending physician.

\section{Endpoints}

The following patient characteristics were assessed: sex, age, height, duration of diabetes, family history, smoking history, alcohol intake history, and complications. We investigated the use of other antidiabetic agents before initiation of sitagliptin, at initiation of sitagliptin, and 12 months after initiation of sitagliptin. The following efficacy endpoints were evaluated at each specified time of assessment: HbA1c (National Glycohemoglobin Standardization Program), blood glucose (fasting/ postprandial), body weight, blood pressure (systolic/diastolic), liver function parameters (glutamic oxaloacetic transaminase, glutamic pyruvic transaminase, and $\gamma$-glutamyl transpeptidase), renal function parameters (serum creatinine and eGFR), serum lipids (total cholesterol, low-density lipoprotein cholesterol, high-density lipoprotein cholesterol, and triglycerides), and serum amylase. In addition, occurrence of AEs was analyzed as the safety endpoint.

\section{Statistical analysis}

Among the registered patients, those included in the present factor analysis received sitagliptin as add-on therapy to insulin, had HbAlc data available up to 12 months, and had no missing explanatory variables.

Appropriate descriptive statistics were calculated for characteristics such as sex, age, and the presence/absence of diabetic complications to compare all patients receiving sitagliptin as add-on therapy to insulin in the ASSIST-K study with the patients enrolled in the present factor analysis. In addition, the number of patients receiving antidiabetic agents (insulin, sulfonylureas, biguanides, thiazolidinediones, $\alpha$-glucosidase inhibitors, and glinides) and the frequency of using each drug class were calculated before add-on sitagliptin therapy, at initiation of sitagliptin, and after 12 months of sitagliptin treatment for the patients included in the present analysis. Descriptive statistics were also calculated for the daily dose of sitagliptin at the start of add-on therapy and after 12 months of treatment.

The values of $\mathrm{HbAl}$ c, body weight, and eGFR measured at the start of add-on sitagliptin therapy and after 3, 6, and 12 months of treatment, as well as changes in these parameters, were displayed in graphs, and pretreatment and post-treatment values were compared by the one-sample $t$-test. The incidence of AEs was calculated during the 12-month sitagliptin treatment period.

Factor analysis was performed by analysis of variance (ANOVA) or with a logistic regression model, using the 12-month changes in HbAlc, body weight, or eGFR and the presence/absence of AEs as the response variables, while sex and age were employed as explanatory variables. The initial multivariable model was established by including all of the explanatory variables showing significance at $\mathrm{P}<0.1$ in univariate analysis. Then the final multivariable model was obtained by stepwise selection at $\mathrm{P}<0.05$.

\section{Results}

\section{Disposition of the subjects}

A total of 1,168 patients were registered, among whom 231 
patients were not using insulin before initiation of sitagliptin therapy. Among the remaining 937 patients, 412 patients were included in the present factor analysis, after excluding 116 patients without sufficient HbA1c data and 409 patients with missing data on explanatory variables.

\section{Subject characteristics}

Table 1 compares the characteristics of all patients receiving sitagliptin as add-on therapy to insulin in the ASSIST-K study with those patients included in the present analysis. All variables showed a similar distribution in the two patient populations, except that no patient had an unknown outcome among those included in the factor analysis.

\section{Use of antidiabetic agents}

Antidiabetic agents used by patients included in the factor analysis are presented in Table 2 . All patients were using insulin before initiation of sitagliptin since this was an enrollment criterion and the mean daily number of insulin injections was 2.7. In addition, the mean number of OHAs prescribed was 1.0 and the most frequent drug was a biguanide (172 patients, $41.7 \%$ ). The mean sitagliptin dose was $46.6 \mathrm{mg}$ at initiation of sitagliptin therapy (baseline), while the mean number of concomitant OHAs decreased slightly to 0.9 . Among concomitant OHAs, a decrease in use by more than $3 \%$ from baseline was noted for thiazolidinediones (use decreased from $11.7 \%$ to $7.0 \%$ ) and $\alpha$-glucosidase inhibitors (use decreased from $24.5 \%$ to $20.1 \%$ ). After 12 months of sitagliptin treatment, the mean dose of sitagliptin was increased to $52.0 \mathrm{mg}$. In contrast, the mean number of concomitant OHAs was reduced to 0.8 , which was slightly lower than at baseline. Among concomitant OHAs, a decrease in use by more than 3\% from baseline was noted for biguanides (use decreased from $38.8 \%$ to $33.0 \%$ ).

\section{Changes in HbA1c, body weight, and eGFR}

Changes in HbA1c over time are displayed in Figure 1. Mean HbA1c decreased from $8.53 \%$ at baseline to $7.86 \%$ after 12 months of sitagliptin treatment. The change in HbA1c over 12 months (mean \pm standard deviation (SD)) was $-0.70 \pm 1.14 \%$, and a significant decrease was noted. On the other hand, mean body weight was the same at baseline and after 12 months (66.4 kg vs. $66.4 \mathrm{~kg}$ ), and the 12-month change in body weight (mean $\pm \mathrm{SD}$ ) was only $-0.04 \pm 3.64 \mathrm{~kg}$, which was not significant (Fig. 2). Unlike body weight, mean eGFR (mL/min/1.73 $\mathrm{m}^{2}$ ) decreased from 75.3 at baseline to $71.6 \mathrm{~mL} / \mathrm{min}$ after 12 months and the 12-month change in GFR (mean \pm SD) was $-4.1 \pm 10.9$, which was a significant decrease (Fig. 3).

\section{Occurrence of AEs}

Table 3 lists the AEs reported up to 12 months after initiation of sitagliptin therapy among the 412 patients included in the factor analysis. AEs comprised severe hypoglycemia in 14 patients $(3.4 \%)$, constipation in 13 patients $(3.2 \%)$, gastrointestinal symptoms in three patients $(0.7 \%)$ and other events in eight patients $(1.9 \%)$.

\section{Factor analysis of 12-month changes in HbA1c, body weight, and eGFR}

The results of factor analysis of the 12-month changes in $\mathrm{HbA1c}$ are listed in Table 4. According to univariate analysis, the factors with a significant influence $(\mathrm{P}<0.10)$ on the change in $\mathrm{HbAlc}$ were baseline dyslipidemia, the duration of diabetes, the baseline daily number of insulin injections, and baseline HbA1c. Multivariate analysis confirmed a significant influence of baseline dyslipidemia and HbAlc. Thus, HbAlc was less likely to be improved by add-on sitagliptin therapy in patients with baseline dyslipidemia and a relatively low baseline HbA1c level.

With regard to the 12-month change in body weight, factors showing a significant influence $(\mathrm{P}<0.10)$ in the univariate analysis were baseline myocardial infarction/angina, baseline hypertension, the baseline daily number of insulin injections, baseline biguanide use, baseline body weight, baseline body mass index (BMI), and baseline HbA1c (Table 5). Multivariate analysis confirmed a significant influence of baseline BMI and $\mathrm{HbAlc}$. In short, patients with a higher baseline BMI tended to lose weight after initiation of add-on sitagliptin therapy, while patients with a higher baseline HbA1c tended to gain weight.

The 12-month changes in eGFR were also subjected to factor analysis (Table 6). According to univariate analysis, the factors with a significant influence on the change in eGFR were baseline myocardial infarction/angina, hypertension, baseline dyslipidemia, the baseline daily number of insulin injections, baseline sulfonylurea use, baseline body weight, baseline BMI, and baseline eGFR. Multivariate analysis confirmed a significant influence of baseline dyslipidemia, sulfonylurea use, body weight, and eGFR. In brief, eGFR showed a smaller decrease in patients with baseline dyslipidemia, those without baseline sulfonylurea use, those with a baseline body weight between $57.2 \mathrm{~kg}$ and $74 \mathrm{~kg}$, and those with a lower baseline eGFR.

\section{Factor analysis of AEs (severe hypoglycemia and constipa- tion)}

The factors showing a significant influence $(\mathrm{P}<0.10)$ on the risk of severe hypoglycemia in univariate analysis were sex and baseline $\mathrm{HbAlc}$. Multivariate analysis confirmed that baseline HbAlc was significant, with severe hypoglycemia being more likely to occur in patients who had a lower baseline HbA1c level (5.60 to $\leq 7.50 \%)$ (Table 7$)$.

When factors with an influence on constipation were assessed, univariate analysis identified a significant influence $(\mathrm{P}<0.10)$ of baseline cerebrovascular disease and baseline 
Table 1. Profile of the Subjects: All Patients Receiving Insulin vs. Patients Included in Factor Analysis

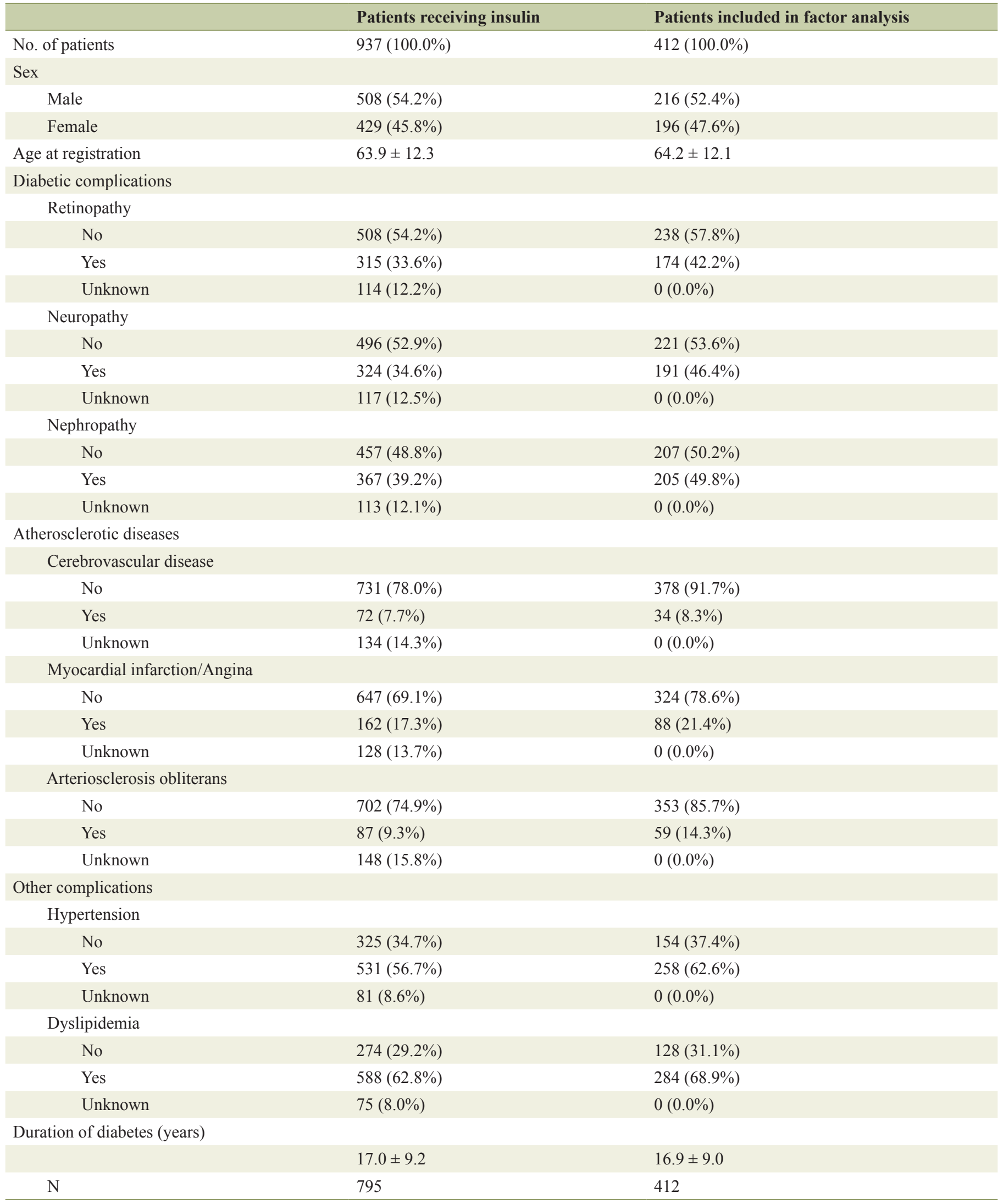


Table 1. Profile of the Subjects: All Patients Receiving Insulin vs. Patients Included in Factor Analysis - (continued)

\begin{tabular}{|c|c|c|}
\hline & Patients receiving insulin & Patients included in factor analysis \\
\hline \multicolumn{3}{|c|}{ Baseline body weight } \\
\hline & $66.23 \pm 15.51$ & $66.36 \pm 14.75$ \\
\hline \multicolumn{3}{|c|}{ Baseline body mass index } \\
\hline & $25.38 \pm 4.61$ & $25.48 \pm 4.40$ \\
\hline & $8.50 \pm 1.35$ & $8.53 \pm 1.35$ \\
\hline $\mathrm{N}$ & 922 & 412 \\
\hline \multicolumn{3}{|c|}{ Baseline eGFR (mL/min/1.73 $\left.\mathrm{m}^{2}\right)$} \\
\hline & $75.9 \pm 22.9$ & $75.3 \pm 22.5$ \\
\hline
\end{tabular}

Men: $194 \times$ serum creatinine $e^{-1.094} \times$ age ${ }^{-0.287}$; Women: $194 \times$ serum creatinine ${ }^{-1.094} \times$ age $^{-0.287} \times 0.739$. Data are shown as the mean \pm standard deviation unless otherwise noted. eGFR: estimated glomerular filtration rate; HbA1c: hemoglobin A1c; NGSP: National Glycohemoglobin Standardization Program.

HbA1c. Multivariate analysis confirmed that baseline HbA1c was significant, with constipation tending to occur in patients who had a lower baseline HbA1c level (5.60 to $\leq 7.50 \%$ ) (Table 8).

Table 2. Use of Antidiabetic Agents

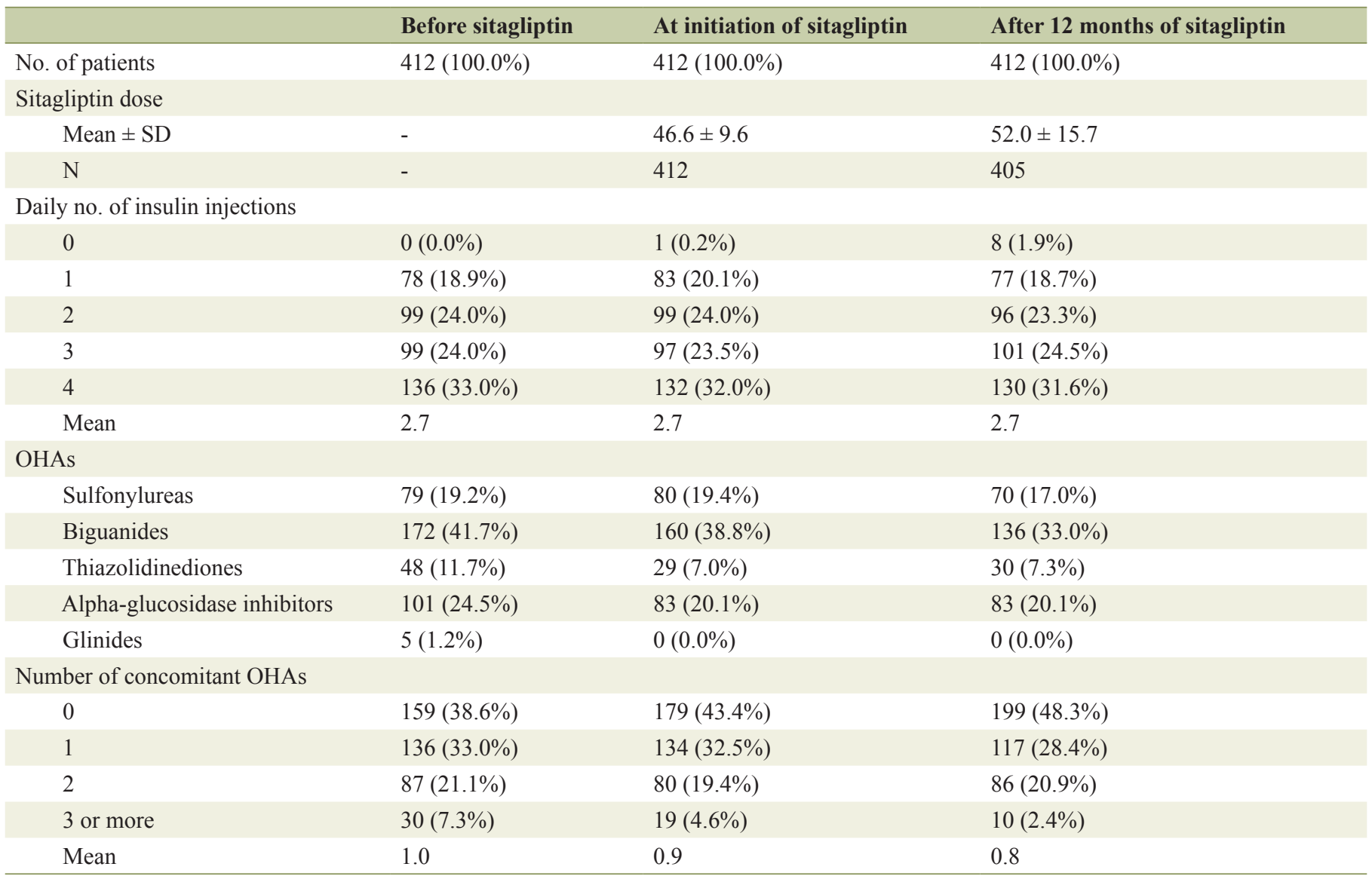

OHA: oral hypoglycemic agent; SD: standard deviation. 

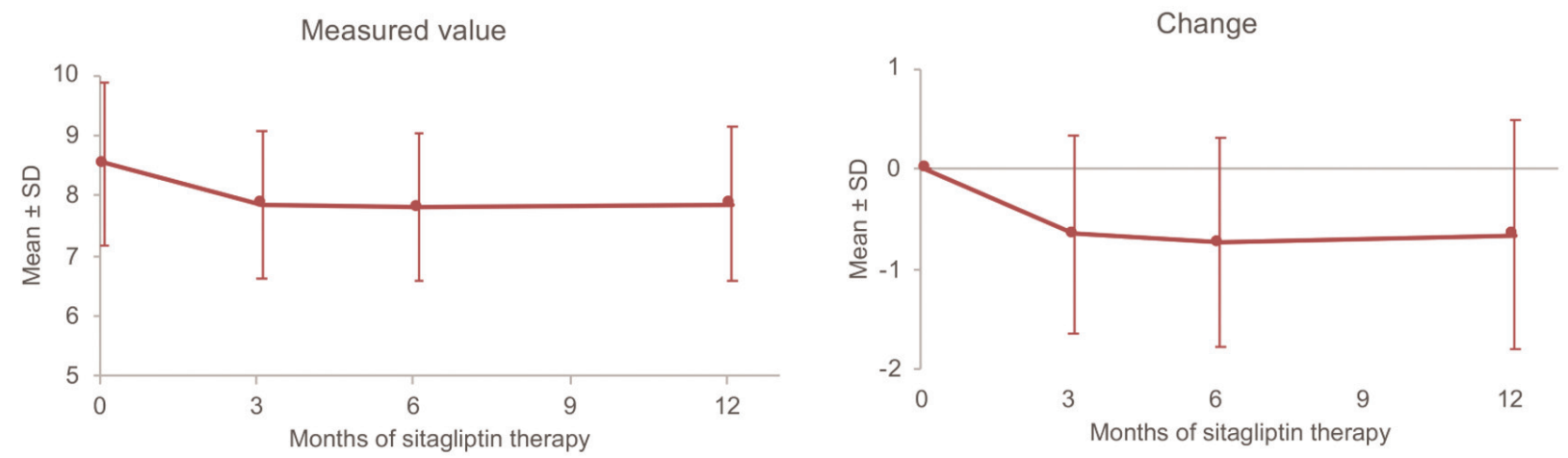

Figure 1. Changes in $\mathrm{HbA} 1 \mathrm{c}$ up to 12 months. SD: standard deviation.
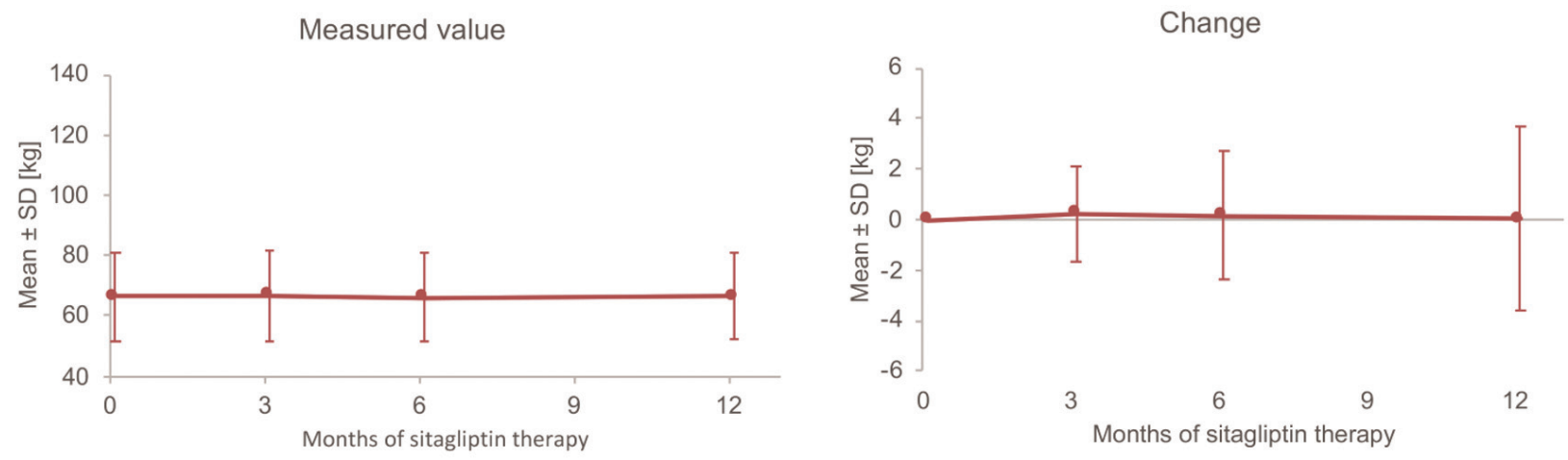

Figure 2. Changes in body weight up to 12 months. SD: standard deviation.
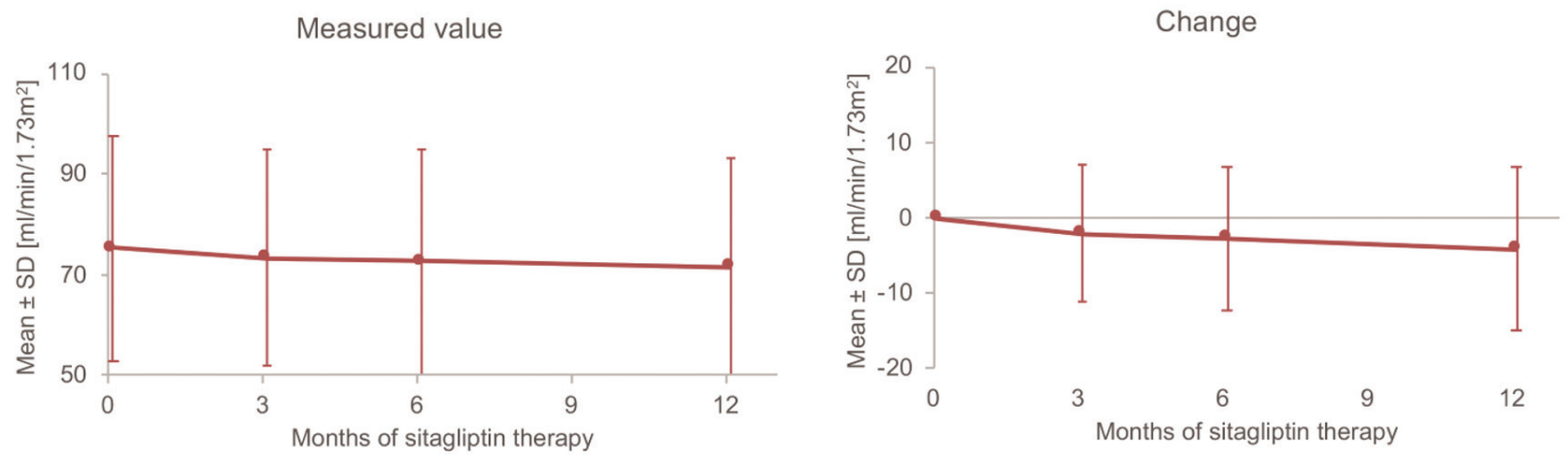

Figure 3. Changes in eGFR up to 12 months. SD: standard deviation.

Table 3. Adverse Events

\begin{tabular}{ll} 
& No. of adverse events (incidence rate), $\mathbf{N}=\mathbf{4 1 2}$ \\
\hline Severe hypoglycemia & $14(3.4 \%)$ \\
Gastrointestinal symptoms & $3(0.7 \%)$ \\
Constipation & $13(3.2 \%)$ \\
Others & $8(1.9 \%)$ \\
\hline
\end{tabular}




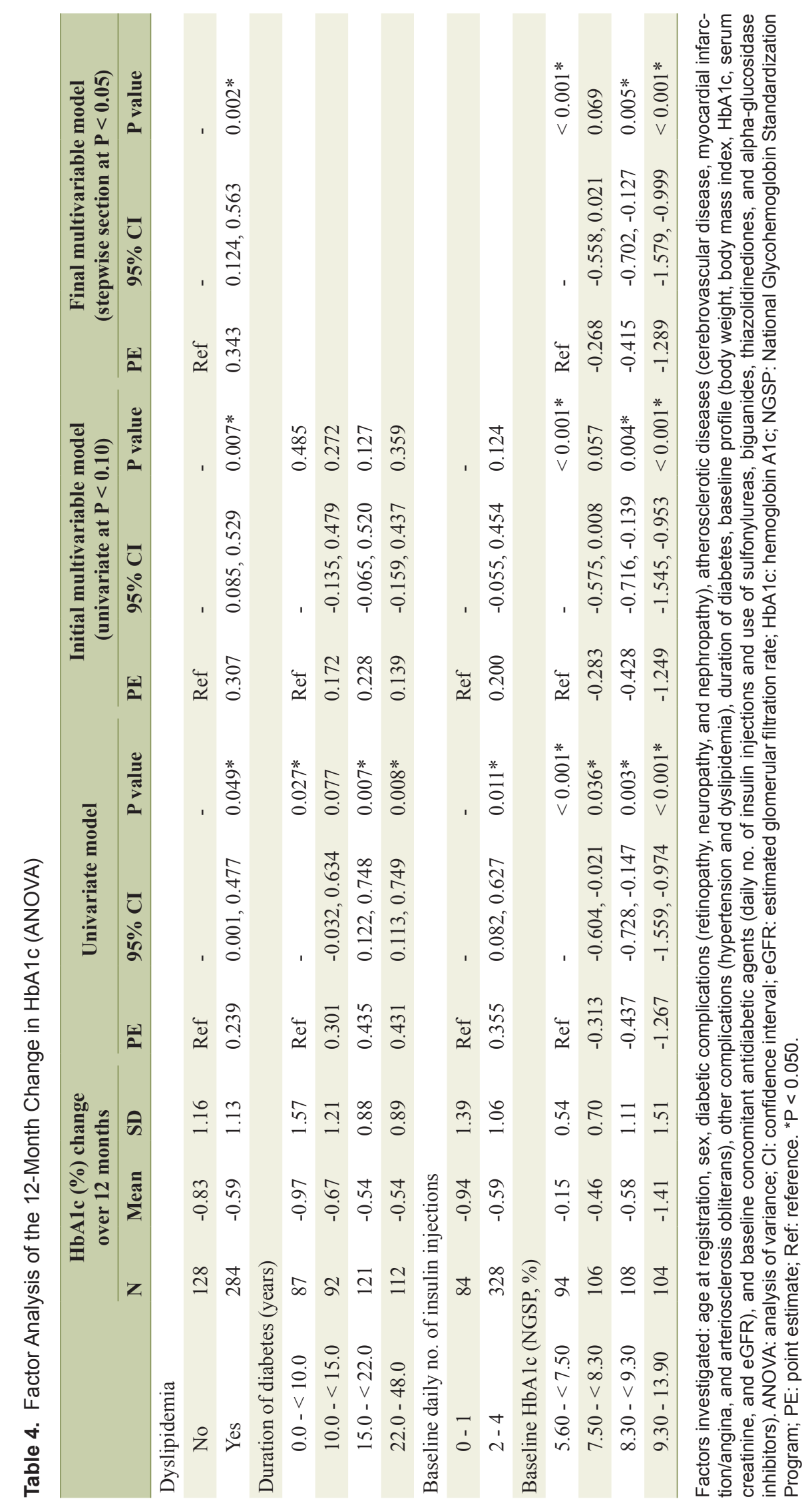




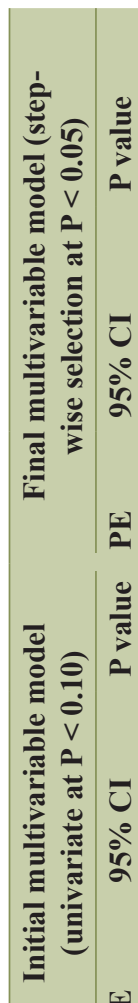

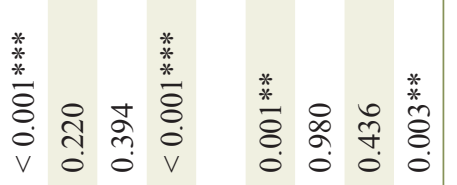

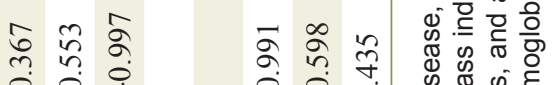

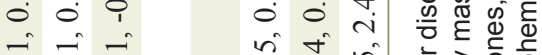

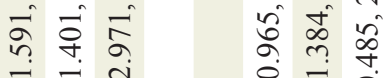

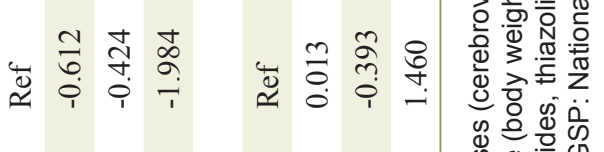

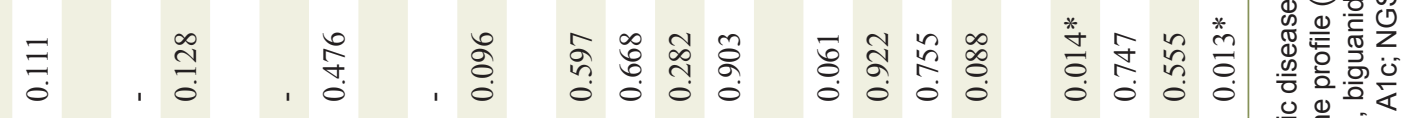

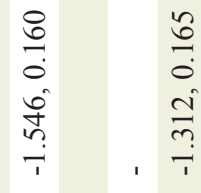

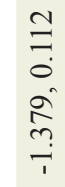

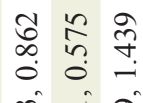

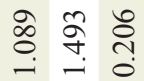

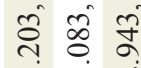

过

O)

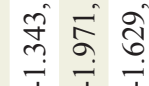

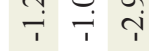

- 2 त

ते ते

,

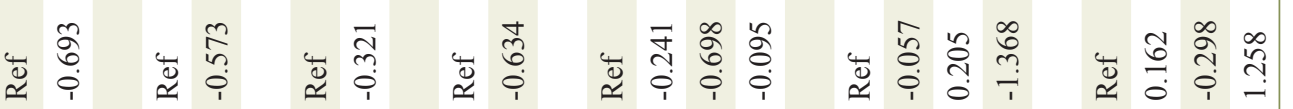

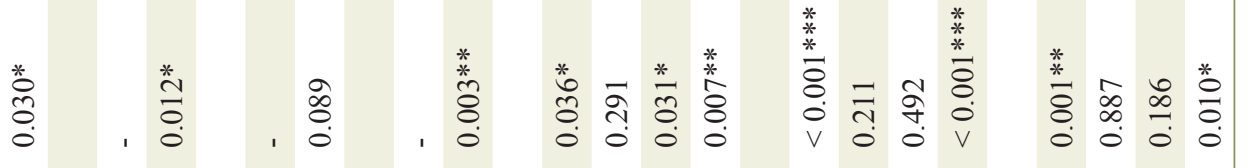

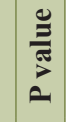

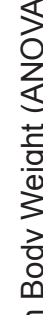

$\stackrel{5}{\mathscr{0}}$

Ix $\stackrel{4}{2}$

胥

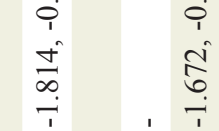

$\begin{array}{ccc}n & n \\ \vdots & 0 \\ 0 & 0 \\ 0 & 0\end{array}$

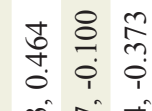

\begin{tabular}{lll}
$\overrightarrow{0}$ & \multirow{1}{*}{} & $\hat{\alpha}$ \\
0 & 0 \\
0 & 0 & 0 \\
0 & 0 & 0
\end{tabular}

ते ले ले है

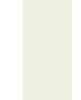

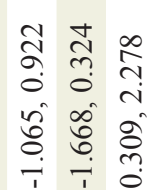

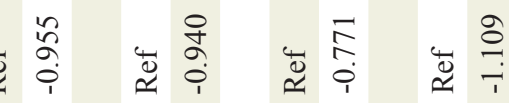

\section{o}

$\rightarrow \rightarrow+$

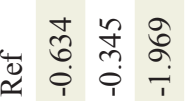

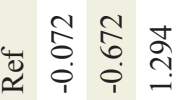

峁

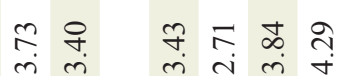

$\because+\frac{1}{2} \approx$

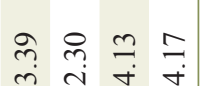

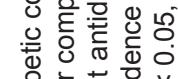

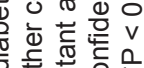

希的记

$\sum_{\substack{i \\ N}}^{\circ}$

길

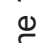

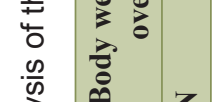

家

产

ए

官

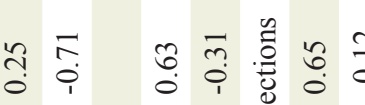

告苍

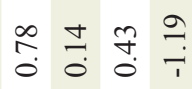

$=\frac{\infty}{i} \stackrel{\infty}{i} \stackrel{\infty}{i} \stackrel{\infty}{=}$

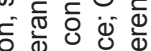

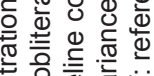

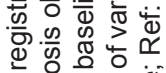

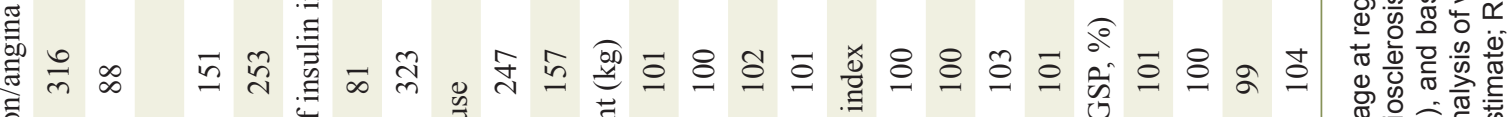

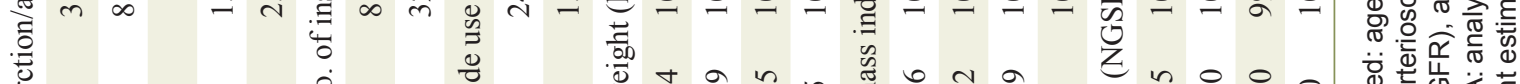

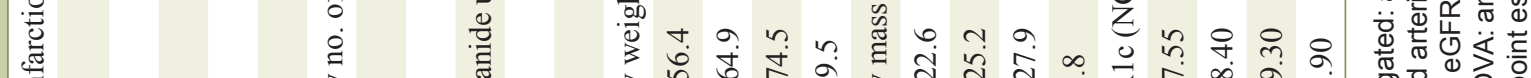

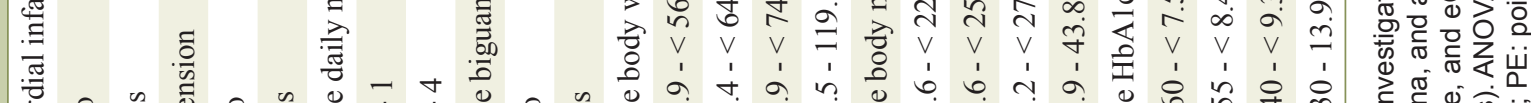

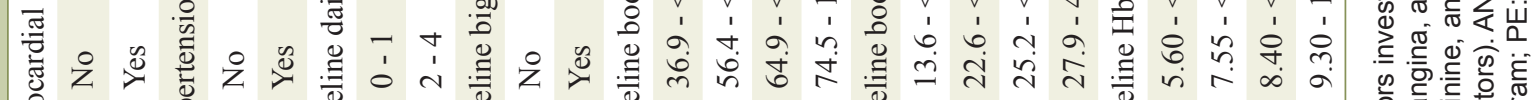

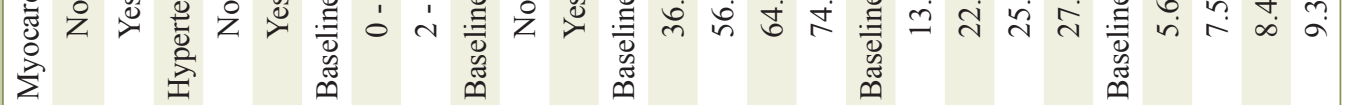




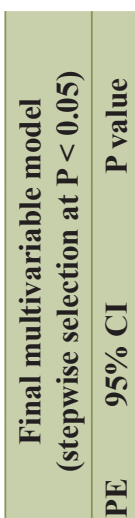

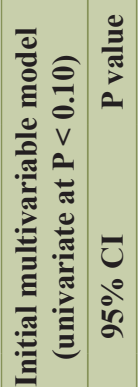

된

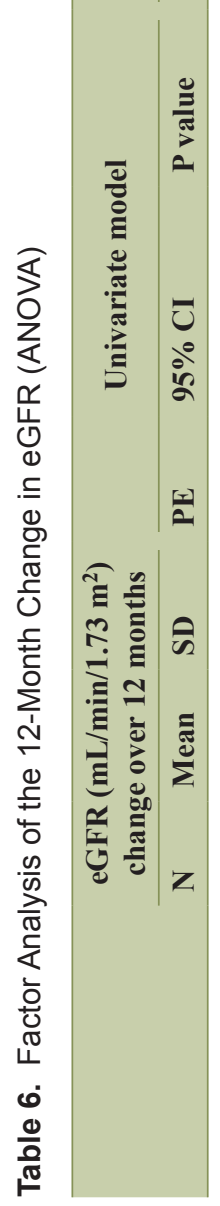

닌

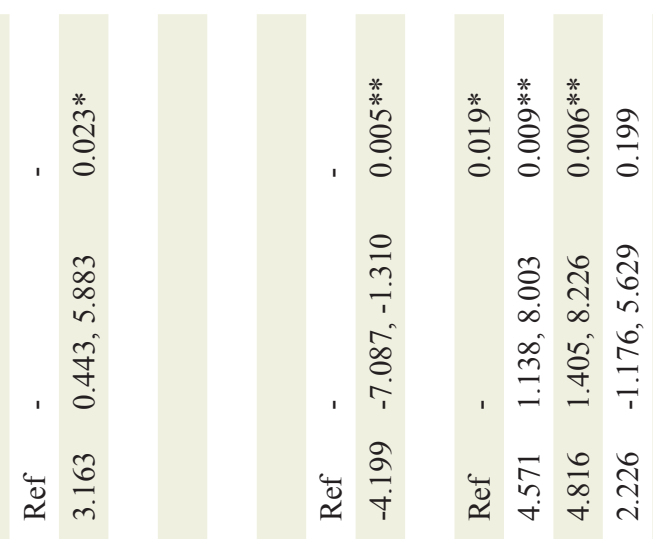

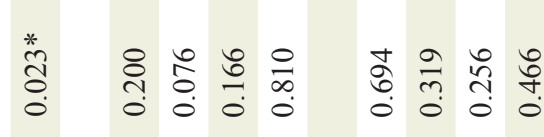

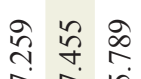

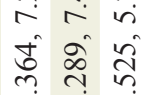

, 空

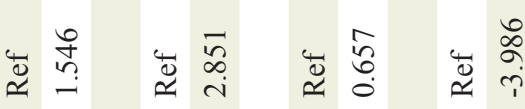

蒡草

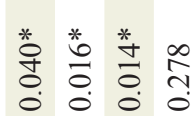

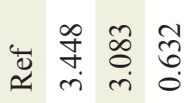

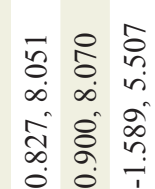

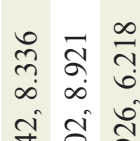

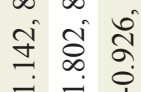

㠃

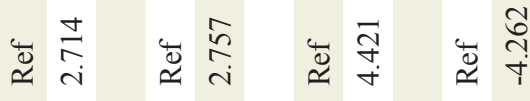

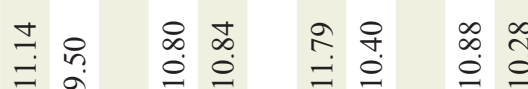

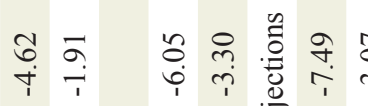

:

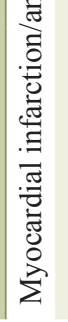

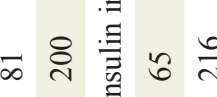

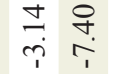

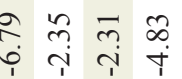

우

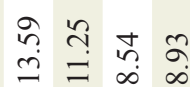

$\stackrel{\circ}{9} \stackrel{\circ}{\circ}:$

in

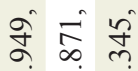

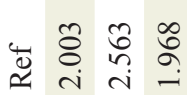

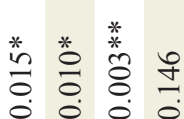

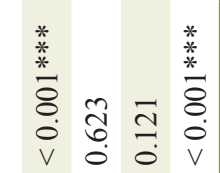
일

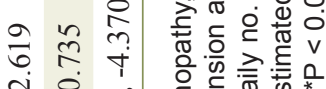

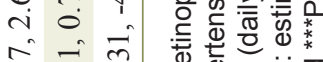

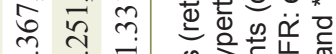
广

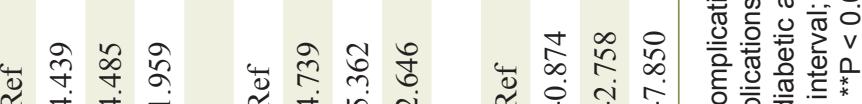
:

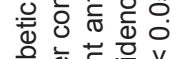

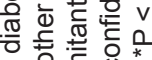

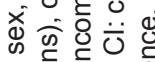
ᄃํ.

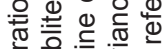

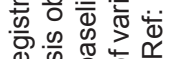

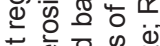

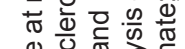

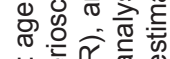

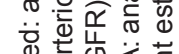

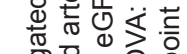

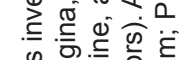
象政 

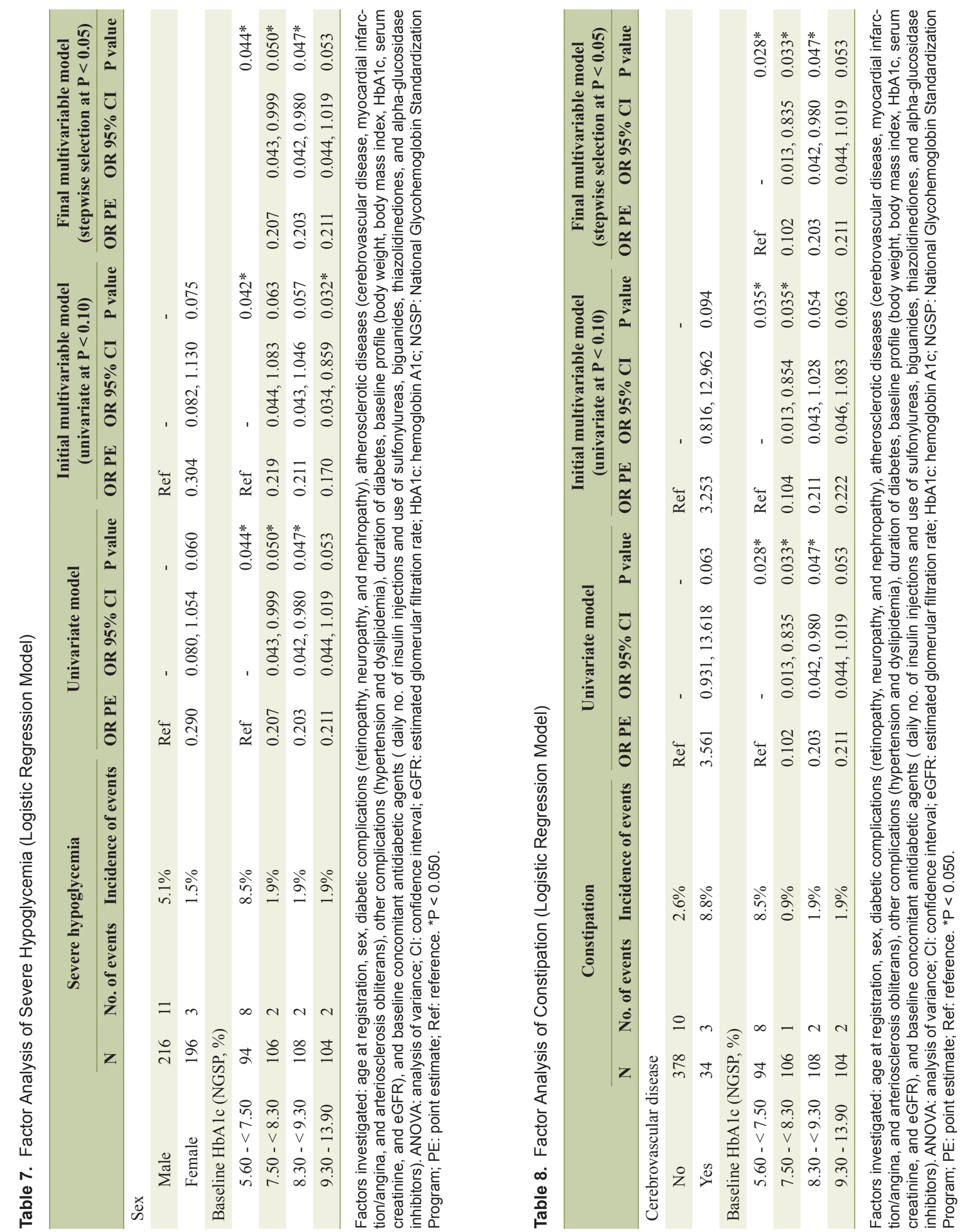


\section{Discussion}

In the present investigation, factor analysis of the 12-month changes in $\mathrm{HbA} 1 \mathrm{c}$, body weight, eGFR, and AEs after initiation of sitagliptin therapy was carried out by using data from the ASSIST-K study, which was performed in patients with type 2 diabetes managed at institutions specializing in diabetes.

Among 937 patients enrolled in the ASSIST-K study who received sitagliptin as add-on therapy to insulin, 412 patients were included in the present factor analysis after excluding those without sufficient $\mathrm{HbA} 1 \mathrm{c}$ data and those with missing explanatory variables. When we compared background factors (age, sex, duration of diabetes, etc.) between the two patient populations, we found there was a similar distribution of all factors assessed and no significant bias due to selection of 412 patients for analysis from the 937 patients enrolled in the ASSIST-K study.

When efficacy endpoints were investigated, $\mathrm{HbAlc}$ and eGFR both decreased significantly over 12 months after initiation of sitagliptin therapy, while body weight showed no significant change. These findings were consistent with the previously reported results of pooled analysis of three studies [19]. Assessment of safety revealed that AEs such as severe hypoglycemia (14 patients, 3.4\%) and constipation (13 patients, $3.2 \%$ ) occurred in more than 10 patients, while the overall results supported the good safety profile of sitagliptin already reported [7-9].

Analysis of factors influencing the change in HbAlc over 12 months suggested that add-on sitagliptin therapy was less likely to achieve a good response of HbAlc in patients with concurrent dyslipidemia and a lower baseline HbA1c level. It has already been reported that $\mathrm{HbA1c}$ is less likely to be reduced by sitagliptin in patients with a low baseline $\mathrm{HbA} 1 \mathrm{c}$ level [20]. On the other hand, although an association between the serum lipid profile and $\mathrm{HbA} \mathrm{c}$ was reported previously [21], no other study has shown that concurrent dyslipidemia may influence the change in HbAlc.

Factor analysis of the change in body weight suggested that patients with weight gain had a lower baseline BMI and lower baseline $\mathrm{HbAlc}$ level. It was previously reported that a low baseline body weight and high baseline HbA1c are associated with weight gain in patients with diabetes $[22,23]$.

Factor analysis of the change in eGFR indicated that patients with a higher eGFR after 12 months of sitagliptin therapy were characterized by concurrent dyslipidemia, no baseline sulfonylurea use, a baseline body weight from 57.2 to $\leq 74.0$ $\mathrm{kg}$, and a lower baseline eGFR. It has already been reported that the decline in eGFR is smaller in patients with a lower baseline eGFR [24], and other studies have suggested that dyslipidemia may be a risk factor for deterioration of renal function [25, 26]. On the other hand, statin therapy (the main treatment for dyslipidemia) has been reported to show a renoprotective effect $[27,28]$. Although the renoprotective effect of statins was not directly evaluated in the present study, our results suggest such a possibility. Since renal dysfunction is a risk factor for hypoglycemia and sulfonylureas are well known to cause hypoglycemia, the use of these drugs tends to be avoided in patients with a low eGFR [29-31]. Our present findings may re- flect such existing clinical circumstances. It was also reported that obesity is a risk factor for renal dysfunction $[25,26]$. In the present study, both patients with a higher body weight and those with a lower body weight tended to have lower eGFR values, but there have been no previous reports of a relationship between low body weight and the change in eGFR.

With regard to AEs, the present study suggested that patients with a lower baseline HbAlc were more likely to develop severe hypoglycemia after initiation of add-on sitagliptin therapy, and it has already been reported that low $\mathrm{HbAlc}$ is a risk factor for hypoglycemia [32].

Our findings also suggested that constipation was more likely to occur in patients with a lower baseline HbA1c level. It has been reported that hyperglycemia is a risk factor for constipation [33], but the opposite result was obtained in this study. Further investigation of this issue will be required because little is known about the risk factors for constipation in patients with diabetes.

The following limitations of the present study need to be considered. First, our factor analysis included less than half of all patients enrolled in the ASSIST-K study (412 vs. 1,168 patients), mainly due to missing information about patient characteristics such as comorbidities. Although comparison of patient characteristics confirmed that the population analyzed was representative, our results should be interpreted in light of this limitation. Second, no information was available about concomitant medications other than antidiabetic agents. It seems likely that many patients received concomitant treatment for dyslipidemia or hypertension, but the effects of these medications could not be assessed by our analysis.

In conclusion, patients with concurrent dyslipidemia and a low baseline HbA1c level were less likely to show a good response of $\mathrm{HbA} 1 \mathrm{c}$ to add-on sitagliptin therapy. Our findings also suggested that AEs (severe hypoglycemia or constipation) were more likely to occur in patients with a low baseline $\mathrm{HbA1c}$.

\section{Acknowledgments}

The authors would like to thank the members of the Kanagawa Physicians Association.

\section{Conflict of Interest}

The authors declare no conflict of interest.

\section{Grant Support}

This study was financially supported by the Kidney Foundation, Japan.

\section{References}

1. Marin-Penalver JJ, Martin-Timon I, Sevillano-Collantes 
C, Del Canizo-Gomez FJ. Update on the treatment of type 2 diabetes mellitus. World J Diabetes. 2016;7(17):354395.

2. Scheen AJ. A review of gliptins for 2014. Expert Opin Pharmacother. 2015;16(1):43-62.

3. Kaku K. First novel once-weekly DPP-4 inhibitor, trelagliptin, for the treatment of type 2 diabetes mellitus. Expert Opin Pharmacother. 2015;16(16):2539-2547.

4. Evans PM, Bain SC. Omarigliptin for the treatment of type 2 diabetes mellitus. Expert Opin Pharmacother. 2016;17(14):1947-1952.

5. Aroda VR, Henry RR, Han J, Huang W, DeYoung MB, Darsow T, Hoogwerf BJ. Efficacy of GLP-1 receptor agonists and DPP-4 inhibitors: meta-analysis and systematic review. Clin Ther. 2012;34(6):1247-1258 e1222.

6. Craddy P, Palin HJ, Johnson KI. Comparative effectiveness of dipeptidylpeptidase- 4 inhibitors in type 2 diabetes: a systematic review and mixed treatment comparison. Diabetes Ther. 2014;5(1):1-41.

7. Scheen AJ. Safety of dipeptidyl peptidase-4 inhibitors for treating type 2 diabetes. Expert Opin Drug Saf. 2015;14(4):505-524.

8. Plosker GL. Sitagliptin: a review of its use in patients with type 2 diabetes mellitus. Drugs. 2014;74(2):223242.

9. Lee M, Rhee MK. Sitagliptin for Type 2 diabetes: a 2015 update. Expert Rev Cardiovasc Ther. 2015;13(6):597-610.

10. Maeda H, Kubota A, Tanaka Y, Terauchi Y, Matsuba I, ASSET-K Study group. The safety, efficacy and predictors for HbA1c reduction of sitagliptin in the treatment of Japanese type 2 diabetes. Diabetes Res Clin Pract. 2012;95(1):e20-22.

11. Kubota A, Maeda H, Kanamori A, Matoba K, Jin Y, Minagawa $\mathrm{F}$, Obana $\mathrm{M}$, et al. Pleiotropic effects of sitagliptin in the treatment of type 2 diabetes mellitus patients. J Clin Med Res. 2012;4(5):309-313.

12. Kubota A, Maeda H, Kanamori A, Matoba K, Jin Y, Minagawa $\mathrm{F}$, Obana $\mathrm{M}$, et al. Efficacy and safety of sitagliptin monotherapy and combination therapy in Japanese type 2 diabetes patients. J Diabetes Investig. 2012;3(6):503-509.

13. Kanamori A, Matsuba I. Factors associated with reduced efficacy of sitagliptin therapy: analysis of 93 patients with type 2 diabetes treated for 1.5 years or longer. J Clin Med Res. 2013;5(3):217-221.

14. Maeda H, Kubota A, Kanamori A, Tanaka Y, Terauchi Y, Matsuba I, ASSET-K Study Group. Long-term efficacy and safety of sitagliptin in the treatment of Japanese Type 2 diabetes (ASSET-K1) to a target of HbA1c $<7 \%$. J Endocrinol Invest. 2013;36(8):568-573.

15. Umezawa S, Kubota A, Maeda H, Kanamori A, Matoba $\mathrm{K}$, Jin $\mathrm{Y}$, Minagawa F, et al. Two-year assessment of the efficacy and safety of sitagliptin in elderly patients with type 2 diabetes: Post hoc analysis of the ASSET-K study. BMC Endocr Disord. 2015;15:34.

16. Takai M, Ishikawa M, Maeda H, Kanamori A, Kubota A, Amemiya H, Iizuka T, et al. Safety and efficacy of adding sitagliptin to insulin in patients with type 2 diabetes: the ASSIST-K study. Diabetes Res Clin Pract. 2014;103(3):e30-33.
17. Maeda H, Kubota A, Kanamori A, Tanaka Y, Terauchi Y, Matsuba I, Study Group of Diabetes Committee KPA. Effects of sitagliptin on the serum creatinine in Japanese type 2 diabetes. Diabetes Res Clin Pract. 2015;108(3):e42-45.

18. Ishikawa M, Takai M, Maeda H, Kanamori A, Kubota A, Amemiya H, Iizuka T, et al. Factors predicting therapeutic efficacy of combination treatment with sitagliptin and insulin in type 2 diabetic patients: The ASSIST-K Study. J Clin Med Res. 2015;7(8):607-612.

19. Yuasa S, Sato K, Takai M, Ishikawa M, Umezawa S, Kubota A, Maeda H, et al. Factor analysis of changes in hemoglobin A1c after 12 months of sitagliptin therapy in patients with type 2 diabetes. J Clin Med Res. 2016;8(6):461-471.

20. Yagi S, Aihara K, Akaike M, Fukuda D, Salim HM, Ishida M, Matsuura T, et al. Predictive Factors for Efficacy of Dipeptidyl Peptidase-4 Inhibitors in Patients with Type 2 Diabetes Mellitus. Diabetes Metab J. 2015;39(4):342347.

21. Thambiah SC, Samsudin IN, George E, Zahari Sham SY, Lee HM, Muhamad MA, Hussei Z, et al. Relationship between dyslipidaemia and glycaemic status in patients with Type 2 diabetes mellitus. Malays J Pathol. 2016;38(2):123-130.

22. Balkau B, Home PD, Vincent M, Marre M, Freemantle N. Factors associated with weight gain in people with type 2 diabetes starting on insulin. Diabetes Care. 2014;37(8):2108-2113.

23. van Dieren S, Czernichow S, Chalmers J, Kengne AP, de Galan BE, Poulter N, Woodward M, et al. Weight changes and their predictors amongst 11140 patients with type 2 diabetes in the ADVANCE trial. Diabetes Obes Metab. 2012;14(5):464-469.

24. Rossing K, Christensen PK, Hovind P, Tarnow L, Rossing $\mathrm{P}$, Parving $\mathrm{HH}$. Progression of nephropathy in type 2 diabetic patients. Kidney Int. 2004;66(4):1596-1605.

25. Dronavalli S, Duka I, Bakris GL. The pathogenesis of diabetic nephropathy. Nat Clin Pract Endocrinol Metab. 2008;4(8):444-452.

26. Gheith O, Farouk N, Nampoory N, Halim MA, AlOtaibi T. Diabetic kidney disease: world wide difference of prevalence and risk factors. J Nephropharmacol. 2016;5(1):49-56.

27. Haynes R, Lewis D, Emberson J, Reith C, Agodoa L, Cass A, Craig JC, et al. Effects of lowering LDL cholesterol on progression of kidney disease. J Am Soc Nephrol. 2014;25(8):1825-1833.

28. Qin X, Dong H, Fang K, Lu F. The effect of statins on renal outcomes in patients with diabetic kidney disease: A systematic review and meta-analysis. Diabetes Metab Res Rev. 2017;33(6):e2901.

29. Alsahli M, Gerich JE. Hypoglycemia in patients with diabetes and renal disease. J Clin Med. 2015;4(5):948-964.

30. Ioannidis I. Diabetes treatment in patients with renal disease: Is the landscape clear enough? World J Diabetes. 2014;5(5):651-658.

31. Deacon CF, Lebovitz HE. Comparative review of dipeptidyl peptidase-4 inhibitors and sulphonylureas. Diabetes Obes Metab. 2016;18(4):333-347. 
32. Lipska KJ, Warton EM, Huang ES, Moffet HH, Inzucchi SE, Krumholz HM, Karter AJ. HbA1c and risk of severe hypoglycemia in type 2 diabetes: the Diabetes and Aging Study. Diabetes Care. 2013;36(11):3535-3542.

33. Ihana-Sugiyama N, Nagata N, Yamamoto-Honda R,
Izawa E, Kajio H, Shimbo T, Kakei M, et al. Constipation, hard stools, fecal urgency, and incomplete evacuation, but not diarrhea is associated with diabetes and its related factors. World J Gastroenterol. 2016;22(11):32523260 . 